FERMILAB-TM-1920

\title{
132 nsec Bunch Spacing in the Tevatron Proton-Antiproton Collider
}

\author{
S. D. Holmes et al. \\ Fermi National Accelerator Laboratory \\ P.O. Box 500, Batavia, Mlinois 60510
}

December 1994 


\section{Disclaimer}

This report was prepared as an account of work sponsored by an agency of the United States Government. Neither the United States Government nor any agency thereof, nor any of their employees, makes any warranty, express or implied, or assumes any legal liability or responsibility for the accuracy, completeness, or usefulness of any information, apparatus, product, or process disclosed, or represents that its use would not infringe privately owned rights. Reference herein to any specific commercial product, process, or service by trade name, trademark, manufacturer, or otherwise, does not necessarily constitute or imply its endorsement, recommendation, or favoring by the United States Government or any agency thereof. The views and opinions of authors expressed herein do not necessarily state or reflect those of the United States Government or any agency thereof. 


\title{
$132 \mathrm{nsec}$ Bunch Spacing in the Tevatron Proton-Antiproton Collider
}

\author{
S.D. Holmes, J. Holt, J.A. Johnstone, J. Marriner, M. Martens, D. $\mathrm{M}^{\mathrm{C}} \mathrm{Ginnis}$ \\ Fermi National Accelerator Laboratory
}

December 23, 1994

\begin{abstract}
Following completion of the Fermilab Main Injector it is expected that the Tevatron proton-antiproton collider will be operating at a luminosity in excess of $5 \times 10^{31} \mathrm{~cm}^{-2} \mathrm{sec}^{-1}$ with 36 proton and antiproton bunches spaced at $396 \mathrm{nsec}$. At this luminosity, each of the experimental detectors will see approximately 1.3 interactions per crossing. Potential improvements to the collider low beta and rf systems could push the luminosity beyond $10 \times 10^{31} \mathrm{~cm}^{-2} \mathrm{sec}^{-1}$, resulting in more than three interactions per crossing if the bunch separation is left unchanged. This paper discusses issues related to moving to $\approx 100$ bunch operation, with bunch spacings of $132 \mathrm{nsec}$, in the Tevatron. Specific scenarios and associated hardware requirements are described.
\end{abstract}




\section{CONTENTS}

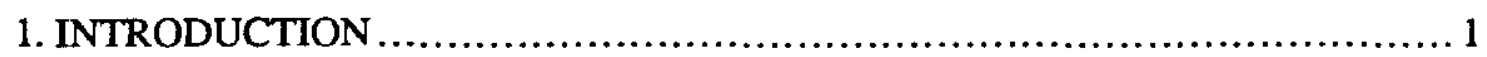

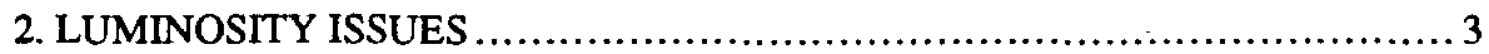

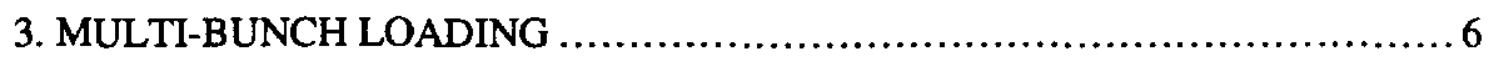

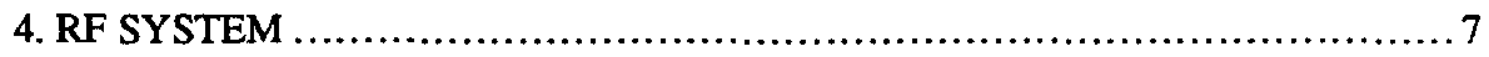

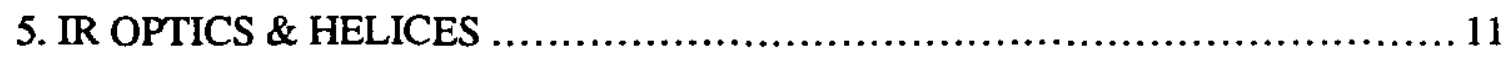

5.1. Dispersion-Free IRs............................................ 11

5.2. Electrostatic Separators \& IP Crossing Angle............................... 16

6. BEAM DYNAMICS ISSUES ............................................ 18

6.1. Crossing Angle ................................................. 18

6.2. Luminosity Lifetime.......................................... 18

6.3. Long Range Beam-Beam Effects ................................. 19

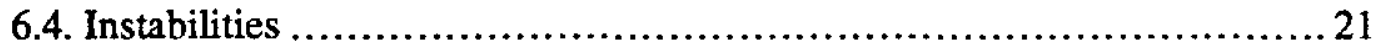

7. SUMMARY OF PERFORMANCE PARAMETERS .......................... 21

7.1. Outstanding Issues and Recommendations for Further Study ............. 23 


\section{INTRODUCTION}

The Tevatron proton-antiproton collider currently operates at $1.8 \mathrm{TeV} / \mathrm{c}$ center-of-momentum, delivering a luminosity greater than $1 \times 10^{31} \mathrm{~cm}^{-2} \mathrm{sec}^{-1}$. This is achieved with six proton and six antiproton bunches colliding at two locations, $\mathrm{B} 0$ (CDF) and D0. The electrostatic separator system causes the two beams to pass with approximately $5 \sigma$ separation on average at the ten other possible collision points around the accelerator. In this configuration each experimental detector, with a sensitivity to about $45 \mathrm{mb}$ of the total $\mathrm{p}-\overline{\mathrm{p}}$ cross section, witnesses 1.6 interactions per crossing.

The Fermilab Main Injector is projected to support a Tevatron luminosity in excess of $5 \times 10^{31} \mathrm{~cm}^{-2} \mathrm{sec}^{-1}$. Hardware currently under construction will allow operation with 36 proton on 36 antiproton bunches when the Main Injector comes on-line in late 1998. A representative set of collider parameters for the first Main Injector-based collider run (Run II) is given in Table 1.1. With this set of parameters CDF and D0 expect to see roughly 2 interactions per crossing. Improvements to the low beta systems, lowering $\beta^{*}$ to $25 \mathrm{~cm}$, and reduction of the rms bunch lengths to $15 \mathrm{~cm}$ or less hold the promise of raising collider luminosity above $10 \times 10^{31} \mathrm{~cm}^{-2} \mathrm{sec}^{-1}$. Continued operation with 36 bunches, however, would result in 3-4 interactions per crossing at this higher luminosity.

This document summarizes a preliminary conceptual design for a Tevatron collider configuration in which bunches are spaced at $132 \mathrm{nsec}$. Increasing the number of bunches is not expected to raise the luminosity: the sole motivation for shorter bunch spacing is to reduce the number of interactions per crossing by about a factor of three, as seen by the detectors. This is seven times the bucket spacing of the current $53.1 \mathrm{MHz}$ Tevatron if system. Multi-bunch schemes with $72,108,96$, and 120 proton and antiproton bunches have been studied for this report.

It is believed that a crossing angle will be required to attain 132 nsec bunch spacing, which dictates that the orbits be separated within the low $\beta$ quadrupoles. The necessary aperture in the low $\beta$ quadrupoles and, conceivably, changes to the low $\beta$ optics which minimize this separation still need to be considered. Also, although long range beam-beam effects are not significant in the current operating mode, once the number of bunches approach 100 the long range effects can no longer be ignored. In particular, effects that need to be considered include bunch-to-bunch tune spreads, bunch-to-bunch closed orbit distortion, and tune spread within a bunch. 
Implementation of any of these multi-bunch scenarios will require new hardware. The introduction of a crossing angle will result in reduced luminosity and the bunch length must be shortened considerably compared to present operations to minimize this impact. This means that a new rf system, operating at $159 \mathrm{MHz}$, will be required. Other new hardware probably includes 1)upgraded low beta optics; 2)upgraded abort kicker; 3) new coalescing cavities operating at three times the frequency $(7.5 \mathrm{MHz}$ ) of those currently operational in the Main Ring and also planned for the Main Injector, and; 4) a new h=12 (7.5 MHz) rf system in the Antiproton Accumulator.

Table 1.1 Collider Parameters for Run II

Protons/bunch

Antiprotons/bunch

Total Antiprotons

Proton emittance (95\%, norm)

Pbar emittance (95\%, norm)

Beta@IP

Beam Energy

Bunches

Longitudinal Emittance (95\%)

if Frequency

if Voltage

Bunch length (rms)

Form Factor

Arc Dipole Field

Circumference

Rev. Frequency

Crossing Half-angle

Form Factor

Typical Luminosity

Integrated Luminosity

Bunch Spacing

Interactions/crossing (@45 mb)

Antiproton tune shift ( 2 crossings)

Proton tune shift ( 2 crossings)

Helix separation $(\sigma)$

Long Range tune spread (antiproton, $2.5 \sigma$ )

\begin{tabular}{|c|c|}
\hline $33 \times 10^{11}$ & \\
\hline $3.6 \times 10^{10}$ & \\
\hline $1.3 \times 10^{12}$ & \\
\hline $30 \pi$ & $\mathrm{mm}-\mathrm{mr}$ \\
\hline $20 \pi$ & $\mathrm{mm}-\mathrm{mr}$ \\
\hline 0.35 & meters \\
\hline 1000 & $\mathrm{GeV}$ \\
\hline 36 & \\
\hline 3 & $\mathrm{eV}-\mathrm{sec}$ \\
\hline 53 & $\mathrm{MHz}$ \\
\hline 1 & MV \\
\hline 0.43 & meters \\
\hline 0.70 & \\
\hline 4.4 & Tesla \\
\hline 6283 & \\
\hline 47.7 & $\mathrm{kHz}$ \\
\hline 0 & $\mathrm{mr}$ \\
\hline 1.00 & \\
\hline $8.3 \times 10^{31}$ & $\mathrm{~cm}^{-2} \mathrm{~s}^{-1}$ \\
\hline 16.7 & $\mathrm{pb}^{-1 /} \mathrm{wk}$ \\
\hline 396 & nsec \\
\hline 2.2 & \\
\hline 0.016 & \\
\hline 0.003 & \\
\hline 5 & \\
\hline 0.008 & \\
\hline
\end{tabular}




\section{LUMINOSITY ISSUES}

The proton-antiproton luminosity in the Tevatron is given by the expression:

$$
L=\frac{\mathrm{fBN}_{\mathrm{p}} \mathrm{N}_{\overline{\mathrm{p}}}}{2 \pi\left(\sigma_{\mathrm{p}}^{2}+\sigma_{\overline{\mathrm{p}}}^{2}\right)} \mathrm{H}\left(\frac{\beta^{*}}{\sigma_{\mathrm{l}}}\right)\left(1+\frac{2 \alpha^{2} \sigma_{\mathrm{l}}^{2}}{\left(\sigma_{\mathrm{p}}^{2}+\sigma_{\overline{\mathrm{p}}}^{2}\right)}\right)^{-1 / 2}
$$

where $f$ is the revolution frequency, $B$ is the number of bunches in each beam, $N_{p}\left(N_{\bar{p}}\right)$ is the number of protons (antiprotons) in a bunch, $\sigma_{\mathrm{p}}\left(\sigma_{\overline{\mathrm{p}}}\right)$ is the transverse rms proton (antiproton) beam size at the interaction point, $\sigma l$ is the rms bunch length, $\beta^{*}$ is the betatron function at the interaction point, and $\alpha$ is the crossing half-angle. $\mathrm{H}$ is the hour-glass form factor:

$$
H(z)=\sqrt{\pi} z e^{z^{2}}[1-\Phi(z)]
$$

with $\Phi(\mathrm{z})$ being the Gauss error function. $\mathrm{H}\left(\beta * / \sigma_{1}\right) \rightarrow 1$ asymptotically, clearly indicating that bunch length should be kept as small as is reasonable compared to $\beta *$ to minimize the luminosity reduction.

The preceding expression for luminosity can be recast slightly into a form that more directly displays limitations inherent to the Tevatron complex:

$$
L=\frac{3 \gamma f N_{p}\left(B N_{\bar{p}}\right)}{\beta^{*} \pi\left(\varepsilon_{N p}+\varepsilon_{N \bar{p}}\right)} H\left(\frac{\beta^{*}}{\sigma_{1}}\right)\left(1+\left(\frac{\alpha \sigma_{1}}{\sigma_{t}}\right)^{2}\right)^{-1 / 2} .
$$

Now $\varepsilon_{N}$ is the normalized (95\%) transverse emittance and $\sigma_{t}$ is the average of the transverse rms $p$ and $\overline{\mathrm{p}}$ beam sizes. Fundamental Tevatron limitations are related to the quantities $\left(\mathrm{N}_{\mathrm{p}} / \varepsilon_{\mathrm{N}}\right)$ and $\left(\mathrm{BN}_{\overline{\mathrm{p}}}\right)$. The first of these is limited by the head-on beam-beam tune shift experienced by the antiprotons, and the second by the total number of available antiprotons. In particular, note that for a fixed number of antiprotons $\left(\mathrm{BN}_{\overline{\mathrm{p}}}\right)$, the luminosity is independent of the number of bunches.

A major limiting factor in the Tevatron proton-antiproton collider is the beam-beam tune shift. In the present collider mode, with six proton and antiproton bunches, there are twelve potential collision points around the ring. Through the use of electrostatic separators the beams can be made to collide with zero crossing angle at the interaction points, yet be separated by $5 \sigma$ (center to center) at the other ten (parasitic) crossings. This basic configuration will be continued with the $36 \times 36$ operation of Run II. However, with 132 nsec bunch spacing this will no longer be possible. 
The nearest separator to the interaction region is beyond the position of the first parasitic crossing for $132 \mathrm{nsec}$ spacing. It does not appear to be possible to avoid these first parasitic collisions unless a crossing angle is introduced to separate the beams within the low $\beta$ quadrupoles. A straightforward calculation ${ }^{1}$ shows that moving the separators upstream of the low $\beta$ triplet, for example, is of little benefit. For realistic gradients and lengths, electrostatic separators are utterly incapable of providing $5 \sigma$ separation at the first parasitic crossing consistent with the $\alpha=0$ constraint at the IP. This assertion remains true largely independent of the value of $\beta^{*}$, and arises primarily because essentially all of the betatron phase advance is generated in the immediate vicinity of the IP ( $45^{\circ}$ of phase advance occurs in $25 \mathrm{~cm}$ for $\beta^{*}=25 \mathrm{~cm}$ for example). An interesting alternative technique for avoiding a crossing angle through the use of rf resonant magnets has been envisioned ${ }^{2}$, but, at least with existing technology, a substantial crossing angle seems to be inescapable.

One major consequence of implementing a crossing angle is to reduce the luminosity by the factor $\left[1+\left(\alpha \sigma_{1} / \sigma_{t}\right)^{2}\right]^{1 / 2}$. Clearly, to minimize luminosity degradation the product of crossing angle and bunch length must be kept as small as possible relative to the transverse beam sizes. The best value for $\alpha$ will be determined based on operating experience, but with a crossing angle of a few hundred $\mu \mathrm{rad}$ the bunch length must be decreased significantly. Since bunch lengths scale as:

$$
\sigma_{1} \propto \frac{\left(\varepsilon_{1}\right)^{\frac{1}{2}}}{\left(\omega_{\mathrm{rf}} V_{\mathrm{rf}}\right)^{\frac{1}{4}}}
$$

a higher frequency rf system will be needed to shorten the bunches.

The length of the luminous region is modified appreciably with the introduction of a crossing angle. Figure 2.1 shows the distribution $\mathrm{dL} / \mathrm{dz}$ (i.e. the vertex distribution that should be seen by an experimental detector) expected for various crossing angles and $14 \mathrm{~cm}$ bunch length. The crossing angle, coupled with the shorter bunch length, results in luminous regions typically of $=8 \mathrm{~cm}$ length (rms) - appreciably shorter than those currently experienced. This feature may have significant consequences for the particle detectors at the interaction regions.

1John A. Johnstone, Report to the Low Beta Study Group I, Internal Fermilab Report, Sept. 29, 1994.

2Gerald P. Jackson, Beam-Beam Collisions of Bunches Separated by $132 \mathrm{nsec}$ without a Crossing Angle, Internal Fermilab Report, Oct. 22, 1994. 


\section{Luminosity Distributions}

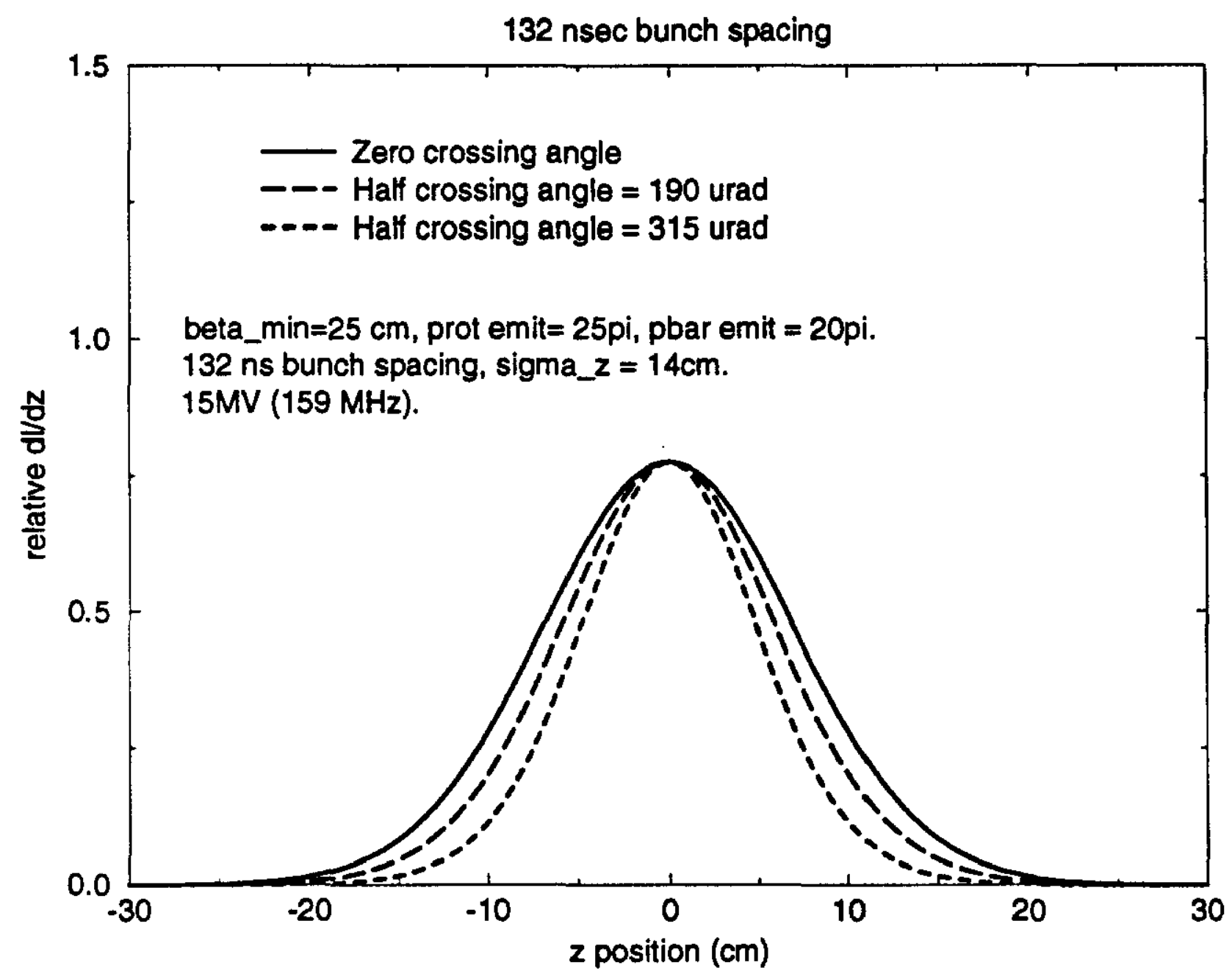

Eigure $2.1 \mathrm{dL} / \mathrm{dz}$ for various crossing angles. 


\section{MULTI-BUNCH LOADING}

The first collider run of the Main Injector era will operate with 36 bunches of protons and antiprotons. A workable configuration calls for three batches of protons and antiprotons containing twelve bunches each, with the batches spaced symmetrically around the ring. The protons would be loaded as three batches of twelve bunches each, with bunches within a batch spaced twenty-one buckets apart (396 nsec). To achieve twenty-one bucket spacing of antiprotons the Accumulator harmonic number must be raised to four. Antiprotons would then be loaded in nine groups of four bunches each. A $\tilde{p}$ injection kicker with a rise time of less than $396 \mathrm{nsec}$ (compared to $900 \mathrm{nsec}$ in $6 \times 6$ operation) and a flattop of at least $1224 \mathrm{nsec}$ (four antiproton bunches twenty-one buckets apart) would be required. The current abort gap of $3.5 \mu \mathrm{sec}$ will shrink to $2.6 \mu \mathrm{sec}$ in $36 \times 36$ operation, requiring modification of the kicker power supply. Installation of the $\bar{p}$ injection kicker and modifications to the abort power supply are planned for the summer of 1995 .

For $132 \mathrm{nsec}$ (seven bucket) bunch spacing the Accumulator harmonic number must increase to twelve. Four constraints were placed on all loading schemes considered:

1. there must be an abort gap and it must be possible to abort both beams cleanly at anytime (there is a short period during cogging, however, when this is not possible);

2. the antiproton injection kicker rise, flattop, and fall times must fit within the abort gap;

3. every antiproton bunch must collide with a proton bunch, and;

4. antiproton bunches are to be injected in groups of twelve -- the most natural configuration.

Two types of loading schemes satisfy all four constraints; a three-fold symmetric scheme with three gaps, and also a one-gap scenario. In both schemes the bunches would be injected into the Tevatron in batches of twelve bunches spaced seven buckets apart, with a minimum batch spacing of twenty-one buckets.

There are two possible three-fold symmetric loading schemes, resulting in either 72 or 108 bunches colliding 3 . In $72 \times 72$ operation two batches of twelve bunches would be spaced twentyone buckets apart, followed by a $3.7 \mu \mathrm{sec}$ gap. This sequence would be repeated twice more around the ring. An abort gap of $3.7 \mu \mathrm{sec}$ is larger than that for $36 \times 36$ operation, and the abort at A0 could be used. The $108 \times 108$ scenario calls for three batches of twelve bunches spaced twentyone buckets apart followed by a gap of $1.8 \mu \mathrm{sec}$. The symmetric loading scheme parameters are summarized in Table 3.1.

$3^{3}$ possible collider mode with $99 \times 99$ bunches has percolated into Fermilab folklore, but such a scheme does not satisfy the fourth constraint and is not considered further here. 
Table 3.1 Multi-Bunch Loading Schemes

\begin{tabular}{|c|c|c|c|}
\hline Crossings & $\begin{array}{c}\text { Harmonic } \\
\text { Number }\end{array}$ & $\begin{array}{c}\text { Abort Gap } \\
(\mu \mathrm{sec})\end{array}$ & $\begin{array}{c}\text { Kicker Rise } \\
(\mathrm{nsec})\end{array}$ \\
\hline 6 & 1 & 3.5 & 900 \\
\hline 36 & 4 & 2.6 & 396 \\
\hline 72 & 12 & 3.7 & 396 \\
\hline 108 & 12 & 1.8 & 396 \\
\hline
\end{tabular}

There are two possible one-gap scenarios, resulting in either $96 \times 96$ or $120 \times 120$ operation. Since the Main Injector will inject into the Tevatron at F0, the abort at $\mathrm{C} 0$ would have to be used during injection. With the bunches cogged into position at $\mathrm{B} 0$, both beams could not be aborted at either $\mathrm{C} 0$ or $\mathrm{A} 0$. If there was only a single experiment, at $\mathrm{D} 0$, then the abort at $\mathrm{A} 0$ could be used.

\section{RF SYSTEM}

Outlined in the following is the voltage per turn necessary to obtain a $15 \mathrm{~cm}$ bunch length at $1 \mathrm{TeV}$ in the Tevatron. The ratio of the beam area to the stationary bucket area is approximately:

$$
\frac{\text { Beam_Area }}{\text { Bucket_Area }}=\frac{\pi}{64} \Delta^{2}\left(1-\frac{5}{384} \Delta^{2}\right)
$$

where $\Delta$ is the total bunch length in radians. Assuming that $95 \%$ of the beam is contained in:

$$
\Delta=2 \sqrt{6} \cdot \omega_{\mathrm{rf}} \cdot \frac{\sigma_{1}}{\mathrm{c}}
$$

where $\sigma_{\mathrm{l}}$ is the rms bunch length, and $\omega_{\mathrm{rf}}=\mathrm{hc} / \mathrm{R}$, with $\mathrm{h}$ being the harmonic number of the rf and $R$ the radius of the Tevatron. The stationary bucket area is given by:

$$
\text { Bucket_Area }=\frac{16}{\omega_{\mathrm{rf}}} \sqrt{\frac{\mathrm{eVE}}{2 \pi \mathrm{h} \eta}}
$$

From the preceding equations the voltage per turn for a given stationary bucket area can be determined: 
Since:

$$
\mathrm{eV}(\text { Bucket_Area, } \mathrm{h})=\left(\frac{\text { Bucket_Area } \cdot \mathrm{c}}{16 \mathrm{R}}\right)^{2} 2 \pi \mathrm{h}^{3} \cdot \frac{\eta}{\mathrm{E}}
$$

$$
\text { Bucket_Area }=\text { Beam_Area }\left(\frac{\text { Beam_Area }}{\text { Bucket_Area }}\right)^{-1}
$$

the voltage per turn as a function of harmonic number, bunch length, and beam area can be determined as shown in Figure 4.1.

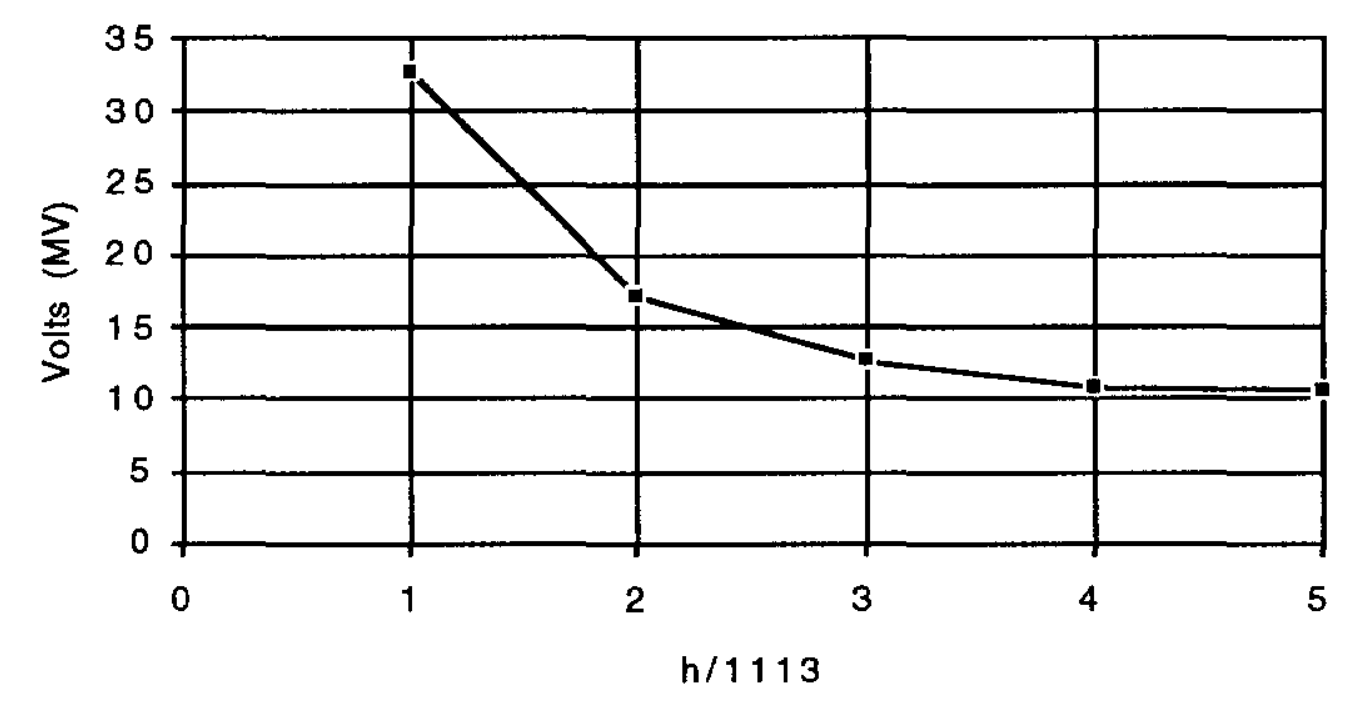

Figure 4.1. The voltage per turn needed for a beam area of $2 \mathrm{eV}-\mathrm{sec}$, and bunch length $\sigma$ of $15 \mathrm{~cm}$ at $1 \mathrm{TeV}$ as a function of the harmonic number of the rf system.

From Fig. 4.1, it is clear that the Tevatron rf system used for collisions should have a frequency at least 3 times greater than the present $53 \mathrm{MHz}$.

Since the Main Injector will have a $53 \mathrm{MHz}$ bunch structure, the rf buckets along some stage of the acceleration chain will have to be switched from the $53 \mathrm{MHz}$ bunch structure to a higher frequency if bucket. The rf voltage per turn needed to provide a $2 \mathrm{eV}$-sec bucket at injection into the Tevatron as a function of the rf system harmonic number is:

$$
\left.\mathrm{V}_{\mathrm{TEV}}\right|_{\mathrm{Inj} .}=231 \mathrm{kV}\left(\frac{\mathrm{h}_{\mathrm{TEV}}}{1113}\right)^{3}
$$


The matching condition between the Main Injector and the Tevatron requires:

$$
\left.\frac{V_{M I}\left(\omega_{M I}\right)^{2}}{h_{M I} \eta_{M I}}\right|_{\text {extraction }}=\left.\frac{v_{\text {TEV }}\left(\omega_{T E V}\right)^{2}}{h_{T E V} \eta_{T E V}}\right|_{\text {injection }}
$$

Combining the last two equations, the rf voltage/turn needed at extraction in the Main Injector is:

$$
\mathrm{V}_{\text {MI }} \mathrm{lextraction}=137 \mathrm{kV}\left(\frac{\mathrm{h}_{\mathrm{TEV}}}{1113}\right)^{4}
$$

The maximum rf voltage that the Main Injector will be able to generate is about $4 \mathrm{MV} / \mathrm{turn}$. For this voltage the maximum hTEV/1113 would be 2.3 . However, a bunch rotation in the Main Injector just prior to extraction could reduce the voltage needed. This rotation voltage is:

$$
\mathrm{V}_{\text {rotation }}=\sqrt{\mathrm{V}_{\text {extraction }} \cdot \mathrm{V}_{\text {min imum }}}
$$

where $\mathrm{V}_{\text {minimum }}$ is the minimum Main Injector voltage needed to provide a $2 \mathrm{eV}$-sec bucket at extraction which is $118 \mathrm{kV} / \mathrm{turn}$. From the above, the voltage is found to be:

$$
\mathrm{V}_{\mathrm{MI}_{\text {ROT }}}=127 \mathrm{kV}\left(\frac{\mathrm{h}_{\mathrm{TEV}}}{1113}\right)^{2}
$$

This final result shows that bunch rotation in the Main Injector could accommodate a Tevatron of frequency up to $5 \times 53 \mathrm{MHz}$.

Figure 4.1 suggests that the if frequency used during collisions should be as high as possible to minimize the if voltage/turn. However, if the same rf system is to be used for acceleration in the Tevatron, then the rf frequency should be as low as possible. Table 4.1 summarizes the possibilities:

\section{Table 4.1.}

\begin{tabular}{|c|c|c|c|}
\hline hTEV/1113 & $\begin{array}{c}\text { VTEV } \\
\text { Collision } \\
\text { (MV) }\end{array}$ & $\begin{array}{c}\text { V TEV } \\
\text { Injection } \\
\text { (MV) }\end{array}$ & $\begin{array}{c}\text { VMI } \\
\text { Rotation } \\
\text { (MV) }\end{array}$ \\
\hline 1 & 32.71 & 0.231 & 0.127 \\
\hline 2 & 17.25 & 1.848 & 0.508 \\
\hline 3 & 12.61 & 6.237 & 1.143 \\
\hline 4 & 10.85 & 14.784 & 2.032 \\
\hline 5 & 10.50 & 28.875 & 3.175 \\
\hline
\end{tabular}


The optimum rf frequency for the Tevatron is 3 times $53 \mathrm{MHz}$, which requires a maximum rf voltage of $12.6 \mathrm{MV} / \mathrm{turn}$.

In the following the amount of power needed at $159 \mathrm{MHz}$ to develop $12 \mathrm{MV} / \mathrm{turn}$ is determined. As a point of reference, Fermilab has a RF cavity built for $159 \mathrm{MHz}$ that was acquired by Fermilab from CERN. This cavity was used as a third harmonic cavity in the Collider Run 1 a. The cavity has the following parameters:

$$
\begin{gathered}
\text { Frequency }=159 \mathrm{MHz} \\
\mathrm{R} / \mathrm{Q}=250 \Omega \\
\mathrm{Q}=40,000 \text { (unloaded) } \\
\text { Maximum voltage }=1 \mathrm{MV} \\
\text { Length }=0.74 \mathrm{~m}
\end{gathered}
$$

The total power dissipated in all of the cavities is:

$$
P_{\text {total }}=\frac{1}{2} \frac{V_{\text {total }^{2}}}{n_{\text {cav }} \frac{R}{Q} Q}
$$

where $\mathrm{n}_{\mathrm{cav}}$ is the total number of cavities. If the total voltage is $12.6 \mathrm{MV}$ and the number of cavities is 12 , so that each cavity develops $1 \mathrm{MV}$, then the total power dissipated in the cavities is $660 \mathrm{~kW}$. Because both protons and antiprotons share the Tevatron there must be two sets of cavities; one set for protons and another for antiprotons. The cavities must be phased so that proton cavities supply no voltage to the antiprotons and the antiproton cavities supply no voltage to the protons. This can only be done by spacing the cavities within a set by an odd multiple of a quarter wavelength. Since a quarter wavelength at $159 \mathrm{MHz}$ is $47 \mathrm{~cm}$ and is shorter than the length of a cavity, the minimum spacing between cavities of a set is $3 / 4$ of a wavelength or $1.41 \mathrm{~m}$. Unfortunately there is not enough room between adjacent cavities of a set to fit another cavity. So the total length of the if section must be:

$$
\mathrm{L}_{\mathrm{RF}}=2 \cdot\left[\frac{3}{4} \frac{\mathrm{c}}{\mathrm{f}_{\mathrm{rf}}}\left(\mathrm{n}_{\mathrm{cav}}-1\right)+\mathrm{L}_{\mathrm{cav}}\right]=16.25 \mathrm{~m} \cdot 2
$$

where the factor 2 results from the separate sets of cavities for protons and antiprotons. 


\section{IR OPTICS \& HELICES}

For purely historical reasons the high gradient ( $140 \mathrm{~T} / \mathrm{m} @ 4.8 \mathrm{kA}$ ) low-beta quadrupoles in the Tevatron are labeled, outward from the IP, consecutively as Q4, Q3, Q2, Q1, Q5, and Q6. These magnets are powered antisymmetrically in pairs across the interaction region so that horizontal and vertical lattice functions become interchanged across the IP. Four independently powered trim quads (70 T/m @ $1.2 \mathrm{kA}$ ) perform the detailed optical match into the regular Tevatron lattice.

\subsection{Dispersion-Free IRs}

In the present mode of Tevatron operation dispersion at the IP $\eta^{*} \approx 0$, but the slope $\eta^{\prime} * \neq 0$. The lattice functions shown in Figure 5.1 illustrate this feature for steps $1 \& 15$ of the current $0.35 \mathrm{~m}$ low-beta squeeze. The non-zero slope $\eta^{\prime}$ results in dispersion reaching its maximum value of the straight within the low-beta triplet - precisely where the beam already reaches its ring-wide maximum from $\beta_{\max } \geq 1 \mathrm{~km}$.

An alternative match to the lattice which gives both $\eta$ and $\eta^{\prime} \equiv 0$ throughout the straight section has been found ${ }^{1}$ which uses the current IR physical configuration of magnets and gradients compatible with the existing quadrupoles. The gradients in the dispersion-free IR scheme at injection and collision are presented in Table 5.1, and the corresponding lattice functions are shown in Figure 5.2 .

Table 5.1. Dispersion-Free $\mathbb{R}$ Gradients $(\mathrm{T} / \mathrm{m}) @ 1 \mathrm{TeV} / \mathrm{c}$

\begin{tabular}{||c||c|c|c|c||c|c||}
\hline \hline Quad \# & \multicolumn{2}{|c|}{$\beta^{*}=3.50 \mathrm{~m}$} & \multicolumn{2}{c||}{$\beta^{*}=0.35 \mathrm{~m}$} & \multicolumn{2}{c||}{$\beta^{*}=0.25 \mathrm{~m}$} \\
& up & down & \multicolumn{2}{c|}{ up } & down & \multicolumn{2}{c|}{ up } & down \\
\hline $\mathrm{Q} 4$ & 144.75 & -144.75 & 131.77 & -131.77 & 136.43 & -136.43 \\
\hline $\mathrm{Q} 3$ & -125.73 & 125.73 & -137.20 & 137.20 & -139.05 & 139.05 \\
\hline $\mathrm{Q}$ & 144.75 & -144.75 & 131.77 & -131.77 & 136.43 & -136.43 \\
\hline $\mathrm{Q} 1$ & -125.60 & 125.60 & 38.85 & -38.85 & 18.49 & -18.49 \\
\hline $\mathrm{Q} 5$ & 103.41 & -103.41 & -139.92 & 139.92 & -186.13 & 186.13 \\
\hline $\mathrm{Q} 6$ & -71.15 & 71.15 & -28.56 & 28.56 & -11.63 & 11.63 \\
\hline $\mathrm{T} 6$ & -2.64 & & -0.47 & & -0.04 & \\
\hline $\mathrm{T} 7$ & 36.33 & -36.73 & 37.60 & -40.34 & 31.92 & -31.33 \\
\hline $\mathrm{T} 9$ & -11.03 & 13.93 & -51.27 & 51.77 & -49.74 & 48.02 \\
\hline $\mathrm{T} 0$ & 9.62 & -7.70 & 4.10 & -14.27 & 8.56 & -14.61 \\
\hline
\end{tabular}



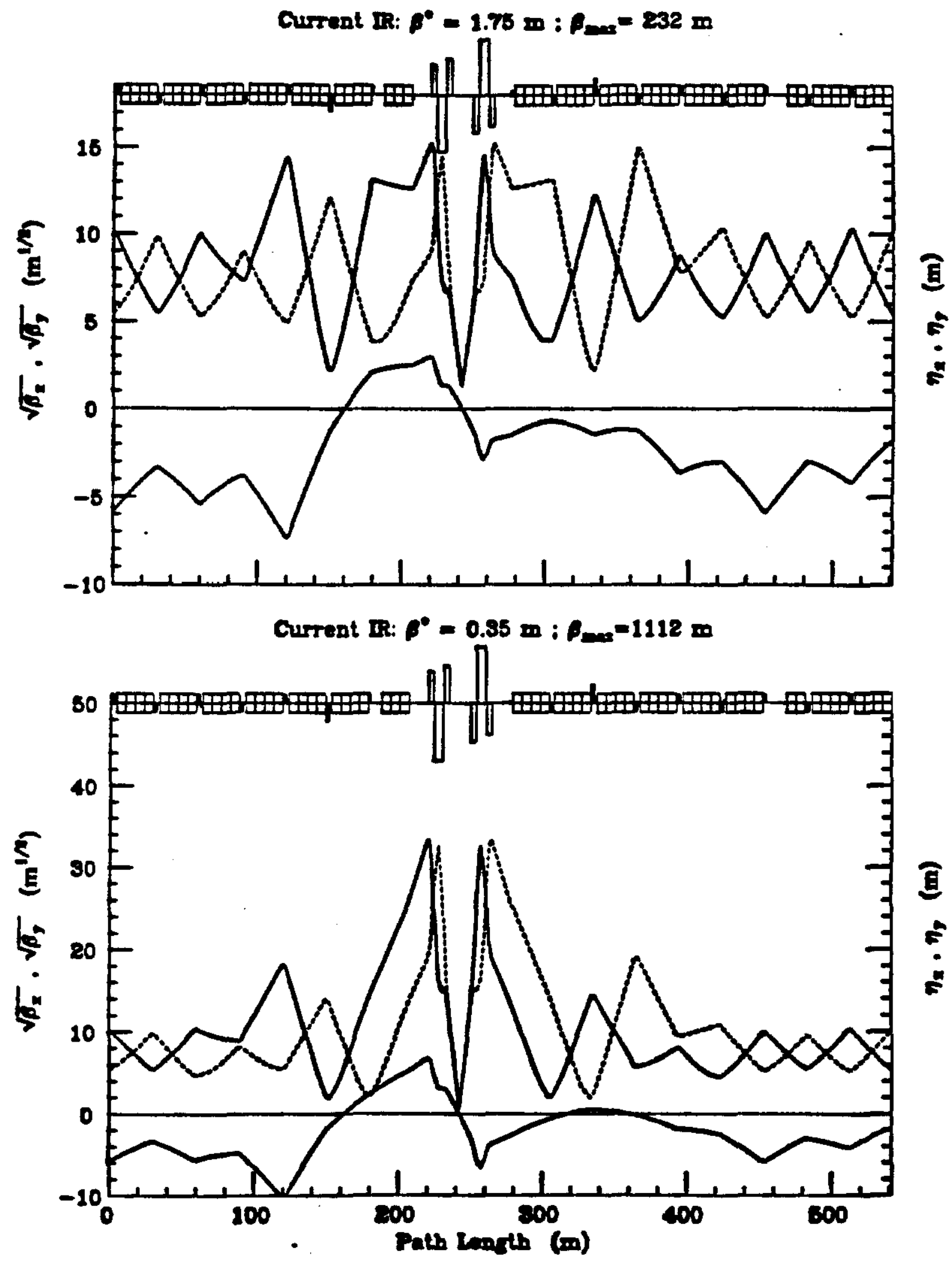

Eigure 5.1. Curment IR Lattice Functions at Injection \& Collision 

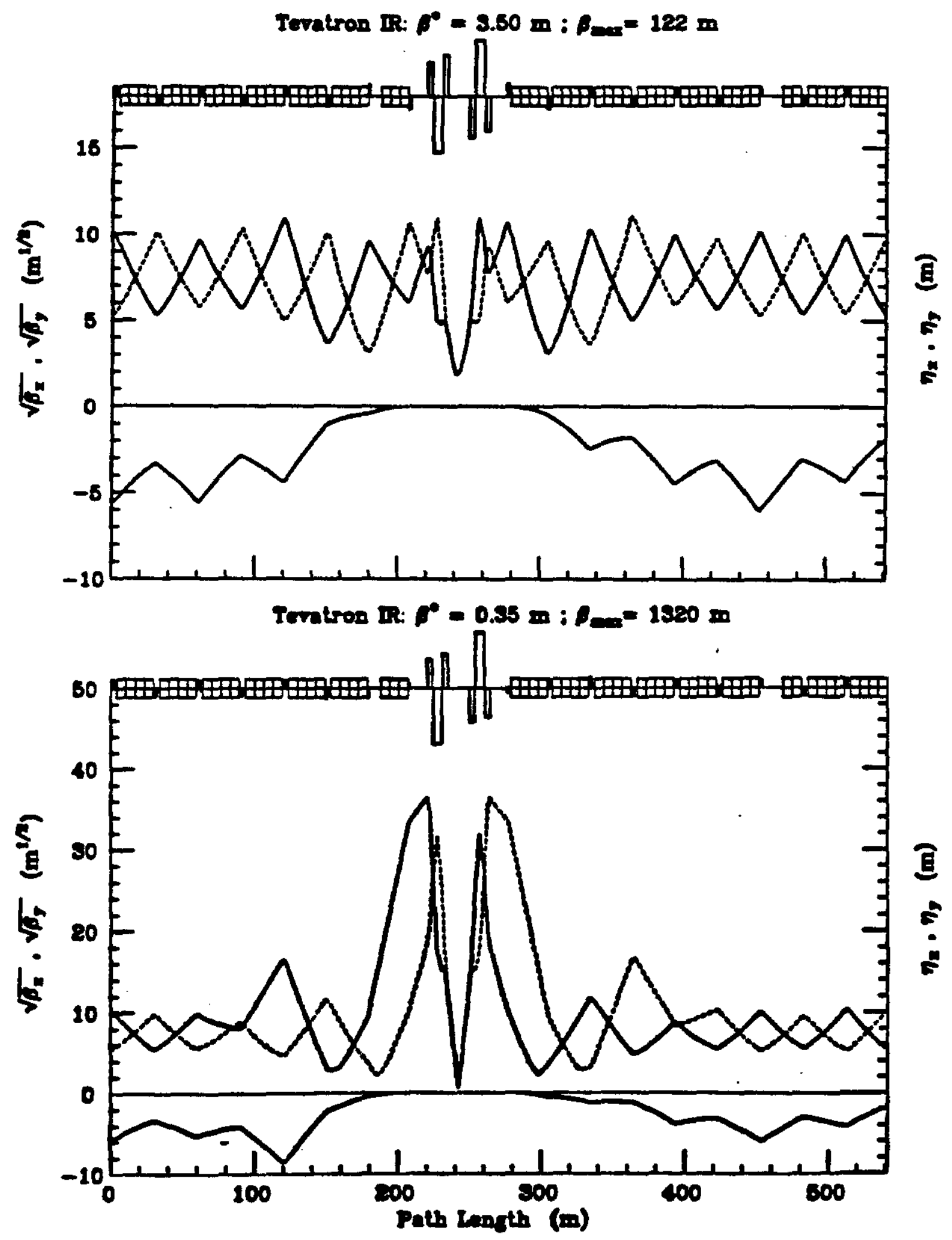

Eigure 5.2Dispersion-Free Lattice Functions at Injection \& Collision 
Although the parameters to achieve $\beta *=0.25 \mathrm{~m}$ are also given in Table 5.1 , it is important to realize that the tabulated $\mathrm{Q} 5$ gradient of $\approx 185 \mathrm{~T} / \mathrm{m}$ is beyond the capabilities of the present system. Implementing the $\beta^{*}=25 \mathrm{~cm}$ solution at $1 \mathrm{TeV} / \mathrm{c}$ would require upgrading to magnets similar to the $250 \mathrm{~T} / \mathrm{m}$ @ 1.8K quadrupoles proposed for CERN's Large Hadron Collider (LHC) ${ }^{4}$.

The variation of the low-beta quadrupole gradients as functions of $\beta *$ for $\beta *=3.50 \mathrm{~m}$ to $0.25 \mathrm{~m}$ are illustrated in Figure 5.3, and the maximum gradients and currents encountered during the $\beta^{*}=35 \mathrm{~cm}$ squeeze are listed in Table 5.2 .

Eigure 5.3. Evolution of Gradients from Iniection to Low Beta

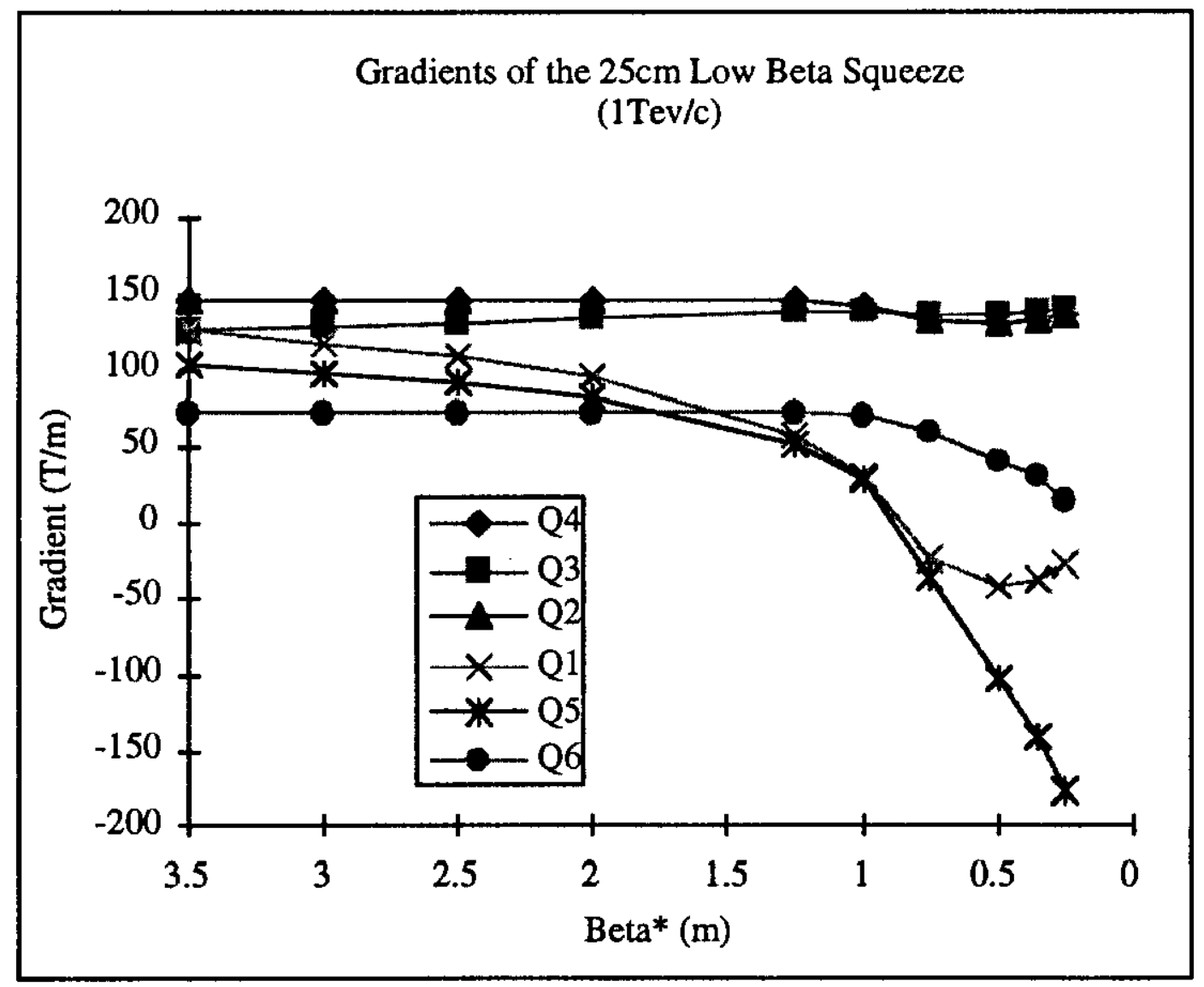

4The LHC Study Group, LHC, The Large Hadron Collider Accelerator Project, CERN/AC/93-03(LHC). 
Table 5.2 Maximum Gradients \& Currents of the Low Beta Squeeze

\begin{tabular}{|}
\hline \multicolumn{3}{|c|}{ Maximum Gradients \& Currents in the 0.35m Low-Beta Squeeze } \\
\hline Quad \# & $\begin{array}{c}\text { Gradient } \\
(\mathrm{T} / \mathrm{m})\end{array}$ & $\begin{array}{c}\text { Current } \\
(\mathrm{kA})\end{array}$ \\
\hline $\mathrm{Q} 4$ & 146.12 & 5.010 \\
\hline $\mathrm{Q} 3$ & 137.20 & 4.704 \\
\hline $\mathrm{Q} 2$ & 146.12 & 5.010 \\
\hline $\mathrm{Q} 1$ & 125.60 & 4.306 \\
\hline $\mathrm{Q} 5$ & 139.92 & 4.797 \\
\hline $\mathrm{Q} 6$ & 71.15 & 2.440 \\
\hline $\mathrm{T} 7$ & 40.54 & 0.692 \\
\hline $\mathrm{T} 9$ & 51.77 & 0.887 \\
\hline T0 & 14.27 & 0.703 \\
\hline
\end{tabular}

The $\beta^{*}=35 \mathrm{~cm}$ solution could be implemented with little modification to the powering of the existing low-beta quads. In the current IR configuration the polarities of quads Q5 and T0 reverse during the squeeze, whereas in the $\eta=\eta^{\prime}=0$ solution it is Q5 and Q1 that reverse. Also, in the proposed scheme the Q1 current goes as high as $4.3 \mathrm{kA}$, while the present Q1 power supply is rated at $2.5 \mathrm{kA}$. One relatively straightforward solution would be simply to use the present Q1 supply to power the Q6 magnets, and the Q6 supply to power the Q1 magnets and reversing switch.

The horizontal beam size with dispersion is given by the rms spread of the Gaussian distribution:

$$
\sigma_{\mathrm{x}}^{2}=\frac{\beta_{\mathrm{x}} \varepsilon_{\mathrm{N}}}{6 \pi \beta \gamma}+\left(\frac{\eta \sigma_{\mathrm{p}}}{\mathrm{p}_{0}}\right)^{2}
$$

where $\varepsilon_{\mathrm{N}}$ is the $(95 \%)$ normalized emittance, $\eta$ is the horizontal dispersion, $\sigma_{\mathrm{p}} / \mathrm{p}_{0}$ is the momentum spread, and $\beta \gamma \approx 1066$ at $1 \mathrm{TeV} / \mathrm{c}$. 
Table 5.3 compares beam sizes in the existing and proposed schemes both at the low-beta quads and in the vicinity of the secondary peaks for $\varepsilon \mathrm{N}=30 \pi \mathrm{mm}-\mathrm{mr}$ and $\sigma_{\mathrm{p}} / \mathrm{p} 0=0.5 \times 10^{-3}$. The dispersion-free solution significantly reduces beam-size in the low-beta quads and at the secondary peaks - particularly at injection. This IR optics configuration is therefore particularly desirable for $132 \mathrm{nsec}$ bunch spacing since the beams must be separated through the IR triplet.

Table 5.3. Beam parameters in the existing \& proposed IR configurations

$$
(\beta *=0.35 \mathrm{~m} @ 1 \mathrm{TeV} / \mathrm{c})
$$

\begin{tabular}{|c|c|c|c|c|c|c|}
\hline \multicolumn{7}{|c|}{ Current IR } \\
\hline $\mathrm{GeV} / \mathrm{c}$ & $\beta_{\max }(\mathrm{T} 7)$ & $\underset{\mathrm{m}}{\eta_{\max }}$ & $\begin{array}{c}\sigma \\
\mathrm{mm}\end{array}$ & $\underset{\mathrm{m}}{\beta_{\max }\left(\mathrm{Q}^{2}\right)}$ & $\underset{\mathrm{m}}{\eta_{\max }}$ & $\begin{array}{c}\sigma \\
\mathrm{mm}\end{array}$ \\
\hline 150 & 198 & 7.33 & 4.43 & 232 & 3.00 & 3.08 \\
\hline 1000 & 290 & 9.00 & 4.65 & 1123 & 4.61 & 3.25 \\
\hline \multicolumn{7}{|c|}{ Dispersion-Free IR } \\
\hline $\begin{array}{c}p \\
\mathrm{GeV} / \mathrm{c}\end{array}$ & $\beta_{\mathrm{m}}^{\max _{2}(\mathrm{~T} 7)}$ & $\underset{\mathrm{m}}{\eta_{\max }}$ & $\begin{array}{c}\sigma \\
\mathrm{mm}\end{array}$ & $\underset{\mathrm{m}}{\beta_{\max }\left(\mathrm{Q}^{2}\right)}$ & $\underset{\mathrm{m}}{\eta_{\max }}$ & $\begin{array}{c}\sigma \\
\mathrm{mm}\end{array}$ \\
\hline 150 & 122 & 4.25 & $\overline{2.26}$ & $12 \overline{2}$ & 0.00 & 1.95 \\
\hline 1000 & 257 & 8.34 & 4.31 & 1320 & 0.00 & 2.49 \\
\hline
\end{tabular}

\subsection{Electrostatic Separators \& IP Crossing Angle}

In order to minimize the degrading effect that a crossing angle has on luminosity a solution has been studied in which $\alpha$ was chosen to be only $190 \mu \mathrm{rad}$, giving $3 \sigma$ separation at the first parasitic crossing. Assuming the current physical location of electrostatic separators, an average of $6.5 \sigma$ separation was maintained at all other parasitic crossings. Table 5.4 lists the voltages at the various separator locations, and the beam-beam separation around the entire ring is illustrated in Figure 5.4. In general the electric fields are comparable to, or less than, those currently in use. The voltages applied at B17 and $\mathrm{C} 17$ in particular are approximately $1 / 4$ of the present values. Further study of the low-beta evolution is required to determine if the number of modules can therefore be reduced. 
Table 5.4. Separator Locations \& Applied Voltages @ $1 \mathrm{TeV} / \mathrm{c}$

\begin{tabular}{|c|c|c|c|c|}
\hline Location & \multicolumn{2}{|c|}{$\begin{array}{c}\text { Horizontal Septa } \\
\text { voltage } \\
(\mathrm{kV})\end{array}$} & \multicolumn{2}{c|}{$\begin{array}{c}\text { Vertical Septa } \\
\text { voltage } \\
(\mathrm{kv})\end{array}$} \\
\hline A17 & & & 1 & -17.59 \\
\hline A49 & 1 & -18.33 & 2 & -19.59 \\
\hline B11 & 2 & -25.00 & 1 & -25.00 \\
\hline B17 & 2 & 4.72 & & \\
\hline C17 & & & 4 & 6.47 \\
\hline C49 & 1 & -24.09 & 2 & -25.59 \\
\hline D11 & 2 & 18.75 & 1 & 25.00 \\
\hline F17 & 1 & -14.15 & & \\
\hline
\end{tabular}

Tevatron : $Q_{\mathrm{r}}=Q_{\mathrm{j}}=20.575: \beta^{\circ}=0.25 \mathrm{~m}$

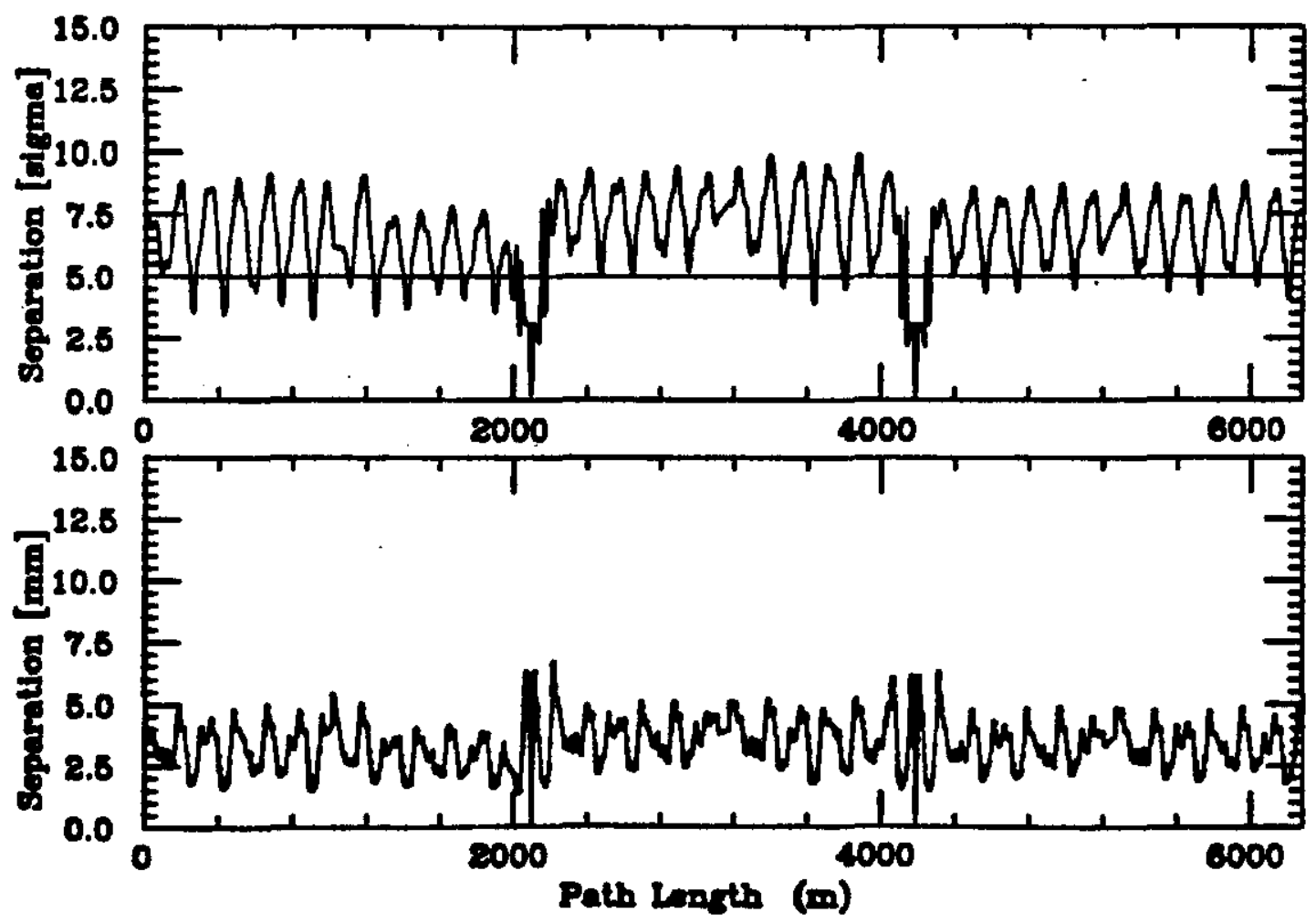

Eigure 5.4. Beam-beam separation around the Tevatron 


\section{BEAM DYNAMICS ISSUES}

\subsection{Crossing Angle}

The primary dynamical consequence of a non-zero crossing angle is thought to be the excitation of synchro-betatron resonances. These resonances were a serious problem at the $e^{+} e^{-}$ collider DORIS 5 . The excitation of such resonances in the Tevatron has not been studied in detail, but it is expected that they will be less important than in the DORIS experience because of the relatively low synchrotron frequency. Note, however, that the proposed parameters and crossing angle for the Tevatron Collider are rather similar to those proposed for the $\mathrm{LHC}^{6}$.

An important consequence of introducing a crossing angle is that the beams will pass through the low-beta quadrupoles off-axis by about $5 \mathrm{~mm}$. The proton and antiproton beams will see slightly different magnetic fields and the focusing fields will be less uniform. While the separated beams may not cause a significant problem, it is worth remarking that the current operating practice in the Tevatron avoids off-center beams at low beta. At the beginning of the low beta squeeze the orbits are separated everywhere (including the interaction regions). During the low beta squeeze, the beams are brought to the centers of the quads when $\beta^{*}$ reaches $0.5 \mathrm{~m}$. The possible effects of synchro-betatron resonances and non-uniformity of the gradient for $132 \mathrm{nsec}$ bunch spacing should be further explored, both theoretically and experimentally.

\subsection{Luminosity Lifetime}

The luminosity lifetime is a major concern because of the setup time involved in each store and because the rate of antiproton production is limited. A model of the luminosity lifetime that includes the effects of the residual gas (nuclear and coulomb scattering), beam-beam collisions, and intrabeam scattering has been developed ${ }^{7}$. This model agrees fairly well with existing Tevatron data except that the longitudinal growth of the proton beam is considerably less than that predicted by intrabeam scattering and the lifetime is less than that predicted from beam-beam collisions. The predictions of this program are shown in Figure 6.1. The program does not include effects from the non-zero crossing angle. Shown separately in Figure 6.1 is the luminosity as a function of time including only the beam loss just from collisions and also the combination of beam-beam collisions and non-zero vacuum.

\footnotetext{
5 A. Piwinski, DESY 77/18, 1977.

6Design Study of the Large Hadron Collider (LHC), CERN 91-03 (2 May 1991).

${ }^{7}$ D. Finley, private communication.
} 


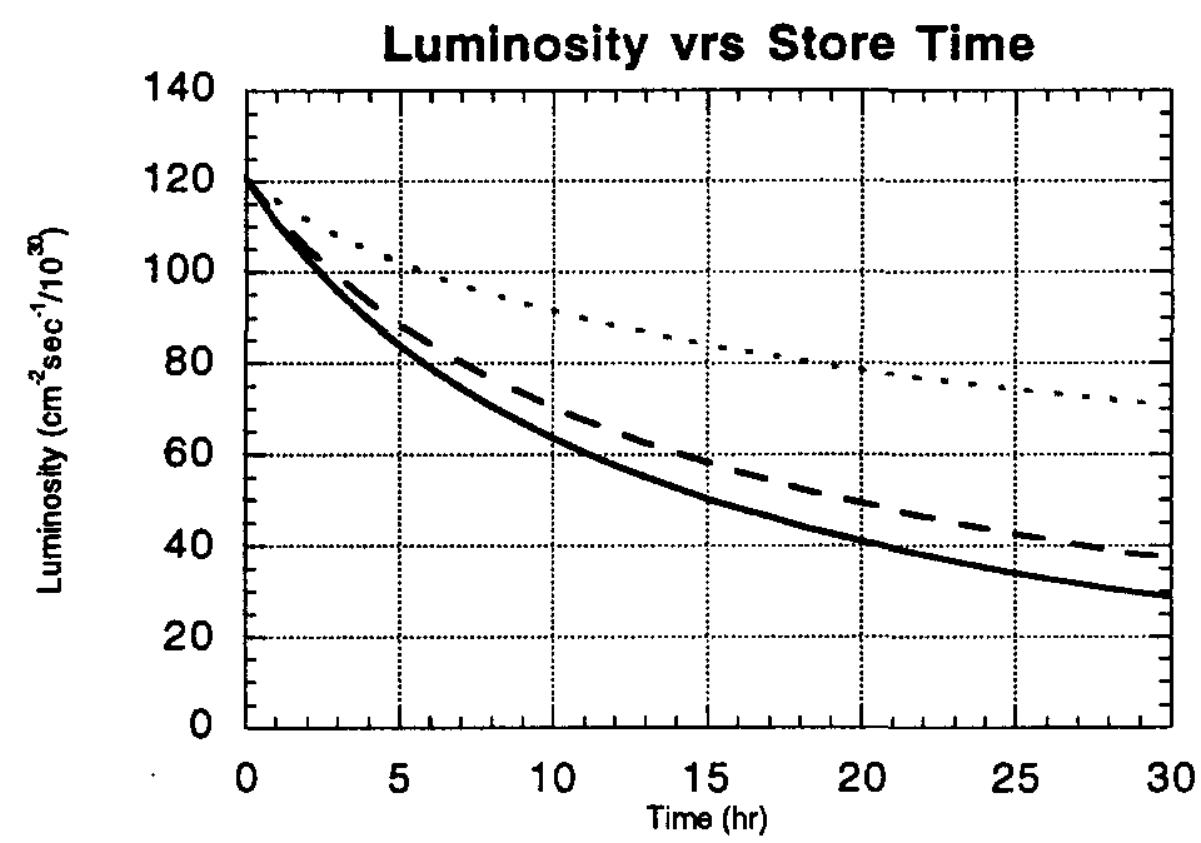

Eigure 6.1 Projected store lifetimes for representative $132 \mathrm{nsec}$ parameters. The dotted curve shows the expected lifetime including only the emittance growth expected from intrabeam scattering. The dashed curve includes the effects of beam-beam collisions, and the solid curve also considers residual gas scattering.

\subsection{Long_Range Beam-Beam Effects}

The large number of parasitic beam-beam crossings leads to significant orbit and tune shifts. If the bunches are not uniformly populated and regularly spaced, each bunch will have a different orbit and a different tune. In the Tevatron the bunches can not be regularly spaced because of the requirement for an abort gap. A bunch loading scheme that leads to 72 bunches colliding with a 132 nsec spacing has been considered. This configuration was chosen because it was thought to be as irregular as any that might be used. The orbit shifts at the interaction points are summarized in Table 6.1. The nominal beam size at the interaction points is $31 \mu \mathrm{m}$, so the maximum orbit shift is about $2 / 3$ of a $\sigma$ of the transverse beam size. The range of tune shifts is shown in Table 6.2. Note that the range of tune shifts is less than, but comparable to, the maximum working space of 0.02 . The range of linear coupling is shown in Table 6.3 and the range of chromaticities is shown in Table 6.4. These effects are not overwhelming, but they are not small. Further study will be required to determine how the effects might be mitigated. 
Table 6.1. Range of Collision Point Offsets for the 72 antiproton bunches

\begin{tabular}{|l|c|}
\hline Direction \& Location & Distance $(\mu \mathrm{m})$ \\
\hline Minimum x at B0 & -20 \\
\hline Maximum x at B0 & -12 \\
\hline Minimum y at B0 & -15 \\
\hline Maximum y at B0 & -11 \\
\hline Minimum x at D0 & -17 \\
\hline Maximum x at D0 & -7 \\
\hline Minimum y at D0 & -8 \\
\hline Maximum y at D0 & 6 \\
\hline
\end{tabular}

Table 6.2. Range of tune shifts for the 72 antiproton bunches

\begin{tabular}{|l|c|}
\hline Tune plane & Tune shift \\
\hline Minimum $\Delta v_{\mathbf{x}}$ & -.0008 \\
\hline Maximum $\Delta v_{\mathbf{x}}$ & .0026 \\
\hline Minimum $\Delta v_{\mathrm{y}}$ & -.0118 \\
\hline Maximum $\Delta v_{\mathrm{y}}$ & -.0017 \\
\hline
\end{tabular}

Table 6.3. Range of coupling shifts for the 72 antiproten bunches

\begin{tabular}{|l|c|}
\hline Coupling type & Coupling shift \\
\hline \hline Minimum $\operatorname{Re}\left(\Delta v_{\mathcal{C}}\right)$ & .0039 \\
\hline Maximum $\operatorname{Re}\left(\Delta v_{\mathcal{C}}\right)$ & .0145 \\
\hline Minimum $\operatorname{Im}\left(\Delta v_{\mathcal{C}}\right)$ &. .0039 \\
\hline Maximum $\operatorname{Im}\left(\Delta v_{\mathcal{C}}\right)$ & .0028 \\
\hline
\end{tabular}

Table 6.4. Range of chromaticity shifts for the 72 antiproton bunches

\begin{tabular}{|c|c|}
\hline Chromaticity plane & Coupling shift \\
\hline Minimum $\xi_{\mathrm{x}}$ & 4 \\
\hline Maximum $\xi_{\mathrm{x}}$ & 22 \\
\hline Minimum $\xi_{\mathrm{y}}$ & -13 \\
\hline Maximum $\xi_{\mathrm{y}}$ & 4 \\
\hline
\end{tabular}




\subsection{Instabilities}

The anticipated $132 \mathrm{nsec}$ beam intensities exceed those currently achieved in the Tevatron and coherent single beam instabilities may result. These might include the transverse head-tail single bunch or coupled bunch instabilities. The head-tail instability is commonly observed if the chromaticity is too low, but can be controlled by active feedback as well as a positive chromaticity. Longitudinal instabilities could include single bunch head-tail modes, coupled bunch, or microwave modes. Single bunch oscillations have been observed in the current collider run, and coupled bunch modes driven by the rf cavities were observed in the last fixed-target run. The single bunch phenomena are not specifically a feature of the $132 \mathrm{nsec}$ spacing but depend on the bunch intensities and emittances. The coupled bunch modes, however, might only be present if large numbers of bunches are present. Possible unstable behavior needs to be studied in more detail and an impedance model of the Tevatron developed.

\section{SUMMARY OF PERFORMANCE PARAMETERS}

A number of scenarios for operation of the Tevatron collider with $132 \mathrm{nsec}$ bunch spacing have been described. Collider parameters are summarized for a representative scenario (108 bunches) in Table 7.1. Three parameter sets are provided for comparison purposes. The left-most column reproduces the projected Main Injector parameter set given in Table 1.1. The middle column represents the projected performance of the collider if the low beta and if improvements described in this report were implemented without proceeding to $132 \mathrm{nsec}$ spacing or introducing a crossing angle. The right hand column represents a possible parameter set utilizing $132 \mathrm{nsec}$ spacing as described in this report.

As indicated in the table, the luminosity in the collider is projected to be approximately $20 \%$ lower in a scenario in which a $132 \mathrm{nsec}$ spacing is produced with a $190 \mu \mathrm{r}$ crossing angle. This results primarily from the loss due to the crossing angle form factor. Other factors, such as reduced proton bunch intensity due to coalescing of fewer bunches, tend to be ameliorated by the accompanying lower longitudinal and transverse emittances. 
Table 7.1: Collider Parameters for 132 nsec Bunch Spacing

\begin{tabular}{|c|c|c|c|}
\hline & $\begin{array}{c}36 \text { bunches } \\
53 \mathrm{MHz} \\
396 \mathrm{nsec}\end{array}$ & $\begin{array}{l}36 \text { bunches } \\
159 \mathrm{MHz} \\
\text { new low } \beta \\
396 \text { nsec }\end{array}$ & $\begin{array}{r}108 \text { bunches } \\
159 \mathrm{MHz} \\
\text { new low } \beta \\
132 \text { nsec }\end{array}$ \\
\hline Protons/bunch & $3.3 \times 10^{11}$ & $3.3 \times 10^{11}$ & $2.7 \times 10^{11}$ \\
\hline Antiprotons/bunch & $3.6 \times 10^{10}$ & $3.6 \times 10^{10}$ & $1.2 \times 10^{10}$ \\
\hline Total Antiprotons & $1.3 \times 10^{12}$ & $1.3 \times 10^{12}$ & $1.3 \times 10^{12}$ \\
\hline Proton emittance $(95 \%$, norm) & $30 \pi$ & $30 \pi$ & $\mathrm{mm}-\mathrm{mr}$ \\
\hline Pbar emittance $(95 \%$, norm) & $20 \pi$ & $20 \pi$ & $\mathrm{mm}-\mathrm{mr}$ \\
\hline Beta @ IP & 0.35 & 0.25 & meters \\
\hline Beam Energy & 1000 & 1000 & 1000 \\
\hline Bunches & 36 & 36 & 99 \\
\hline Longitudinal Emittance $(95 \%)$ & 3 & 3 & eV-sec \\
\hline rf Frequency & 53 & 159 & $\mathbf{M H z}$ \\
\hline If Voltage & 1 & 15 & MV \\
\hline Bunch length (rms) & 0.43 & 0.17 & meters \\
\hline Hour-Glass Form Factor & 0.70 & 0.86 & 0.89 \\
\hline Arc Dipole Field & 4.4 & 4.4 & Tesla \\
\hline Circumference & 6283.0 & 6283.0 & 6283.0 \\
\hline Rev. Frequency & 47.71 & 47.71 & 47.71 \\
\hline Crossing Half-angle & 0 & 0 & 0.19 \\
\hline Crossing Angle Form Factor & 1.00 & 1.00 & 0.77 \\
\hline Typical Luminosity & $8.3 \times 10^{3} 1$ & $14.2 \times 10^{31}$ & $10.4 \times 10^{31} \mathrm{~cm}^{-2} \mathrm{~s}^{-1}$ \\
\hline Integrated Luminosity & 16.72 & 28.67 & $20.99 \mathrm{pb}^{-1 / \mathrm{wk}}$ \\
\hline Bunch Spacing & 396 & 396 & 132 nsec \\
\hline Interactions/crossing (@45 mb) & 2.17 & 3.73 & 0.91 \\
\hline Antiproton tune shift ( 2 crossings) & 0.016 & 0.016 & 0.016 \\
\hline Proton tune shift ( 2 crossings) & 0.003 & 0.003 & 0.001 \\
\hline Helix separation (sigma) & 5 & 5 & 6.5 \\
\hline Separation at first crossing $(\mathrm{d} / \sigma)$ & 5 & 5 & 3.04 \\
\hline Long Range tune spread (antiproton, $2.5 \sigma$ ) & 0.008 & 0.008 & 0.008 \\
\hline
\end{tabular}




\subsection{Outstanding Issues and Recommendations for Further Study}

This report represents a concept for a particular implementation of approximately 100 bunches in the Tevatron Collider. It is not a complete design study. Many of the issues related to increasing the number of bunches have been looked at and potential directions for addressing these issues have been described. Almost all these issues need further study before real engineering designs can be initiated. Important issues in this category are:

1. Can separation at the first parasitic crossing be provided without use of a crossing angle?

2. The anticipated longitudinal emittance needs to be understood. It impacts directly on if requirements and on expectations for the bunch lengths.

3. The implications of $159 \mathrm{MHz}$ for fixed-target operation should be determined.

4. The rf cavity spacing needs to be re-examined to determine if it is optimized.

5. Details of the rf system need to be worked out.

6. A careful analysis of the aperture availability, dynamic and physical, needs to be undertaken for the increased momentum spread associated with shorter bunches, and for the specific helix and low beta designs being considered.

7. Further work should be devoted to improving the low beta optics.

8. Long range beam-beam effects need to be studied both experimentally and through simulation.

9. Intrabeam scattering needs to be carefully calculated.

10.Beam stability with the listed parameters needs to be studied both experimentally and theoretically.

11. Beam loading compensation needs to be understood for the higher average currents projected.

12.The selection of proton and antiproton parameters should be re-examined to see if they are optimized. In particular, it may be desirable to try to run with a lower proton emittance than indicated. 